\title{
Exploration of energy levels using diffraction gratings
}

Guillermo Ezequiel Sánchez-Guerrero, Jose Ismael Martínez-Contreras, Guadalupe Ponce-Hernández, Perla Marlene Viera-González

Guillermo Ezequiel Sánchez-Guerrero, Jose Ismael Martínez-Contreras, Guadalupe Ponce-Hernández, Perla Marlene Viera-González, "Exploration of energy levels using diffraction gratings," Proc. SPIE 11143, Fifteenth Conference on Education and Training in Optics and Photonics: ETOP 2019, 111433P (2 July 2019); doi: 10.1117/12.2523851

Event: Fifteenth Conference on Education and Training in Optics and Photonics: ETOP 2019, 2019, Quebec City, Quebec, Canada 


\title{
Exploration of energy levels using diffraction gratings
}

\author{
Guillermo Ezequiel Sánchez-Guerrero ${ }^{\mathrm{a}}$, Jose Ismael Martínez-Contreras ${ }^{\mathrm{a}}$, Guadalupe \\ Ponce-Hernández ${ }^{\mathrm{a}}$, and Perla Marlene Viera-González ${ }^{\mathrm{a}}$ \\ ${ }^{a}$ Facultad de Ciencias Fisico Matematicas, Universidad Autonoma de Nuevo Leon, Av. \\ Universidad SN Cd. Universitaria, San Nicolas de los Garza, Nuevo Leon, Mexico
}

\begin{abstract}
The electromagnetic spectrum is usually classified by its wavelength and the energy levels associated with it. Teaching these relations to kids without an exploration activity could be a real challenge. On this subject, we have designed a lesson using lasers, diffractions gratings, and photoluminescent materials. This lesson includes activities that allow introducing different optics and photonics concepts. The warm-up activities are to learn the monochromatic nature of lasers, show how diffractions gratings work and explain the concepts of phosphorescence and fluorescence. All this knowledge is combined to see the different energy levels associated with three colors of lasers: red, green and violet. Using different lasers the kids explore which ones activate the fluorescence or phosphoresce effects in the materials provided and, finally, they examine these effects using diffraction gratings. The diffraction gratings allow exploring how a laser can cause fluorescence and how the light emitted by this phenomenon is related to the color of the laser and other colors with less energetic wavelengths. Also, the different lasers are used to analyze which one can cause phosphoresce over materials and comparing the violet lasers with the UV light. In the end, the conclusion is based on the law of conservation of energy.
\end{abstract}

Keywords: Outreach, Education, Diffraction gratings

\section{INTRODUCTION}

The wavelength of the electromagnetic wave usually classifies the electromagnetic spectrum, and visible light is a fraction of it that human eyes can detect. ${ }^{1}$ The visible region can be seen in the table 1 , each subregion has a color associated with it. Because the electromagnetic wave energy is $E=h c / \lambda$ where $h$ is Planck's constant, $c$ is the speed of light and $\lambda$ is the wavelength, the table 1 is sorted from higher energies to lower energies.

Table 1. Colors are associated to a region of the electromagnetic spectrum.

\begin{tabular}{|c|l|}
\hline Wavelength range $(\mathrm{nm})$ & Subregion \\
\hline $380-430$ & Violet \\
\hline $430-500$ & Blue \\
\hline $500-520$ & Cyan \\
\hline $520-565$ & Green \\
\hline $565-580$ & Yellow \\
\hline $580-625$ & Orange \\
\hline $625-740$ & Red \\
\hline
\end{tabular}

Although this is a fundamental concept, teaching it to kids can be difficult, this work presents a series of activities presented in schools of Nuevo León, México by the group Física Pato2 and through the program Optics 4 every $1^{2,3}$ from the Facultad de Ciencias Físico Matemáticas. It contains a general series of how the lesson is presented, but it will keep improving as feedback is received. The objective of the lesson is to manage misconceptions and present a new concept to kids by the exploration of phenomena.

Further author information: (Send correspondence to G.E.S.G.)

G.E.S.G.: E-mail: perla.vieragn(at)uanl.edu.mx 


\subsection{Diffraction gratings}

Diffraction gratings or diffraction glasses are widely used in outreach activities, and they amaze kids and can show different properties of light. The most common use is to separate white light in all the subregions of the electromagnetic spectrum and can be done just by using the diffraction lenses under daylight but never looking directly into it. Usually, the first time a kid uses the glasses, it takes time to let them explore and understand what they are looking. This first exploration is beneficial in outreach activities because it engages the kids with the activity, also is the first step for the lesson, learn how to use the material. This first exploration usually leads to questions from the kids, and sometimes they want to experiment with other light sources like a cellphone. If sunlight is not available, a source like a LED can be used and produce a similar effect. The lenses and a white light source can be seen in the top pictures of Figure 1.

Diffraction glasses can be combined with other sources; in this activity the source is a Laser pointer, point it to a screen, never to the eyes, to show the monochromatic nature of the source. This experience is different from the white source, and the diffraction glasses only show a monochromatic grid of laser color points. This exploration is preferably using three different sources, red, green and violet lasers, this is due to that they will see a full separation of colors and not a continuum like with white sources. This can be seen in the bottom pictures of Figure 1.
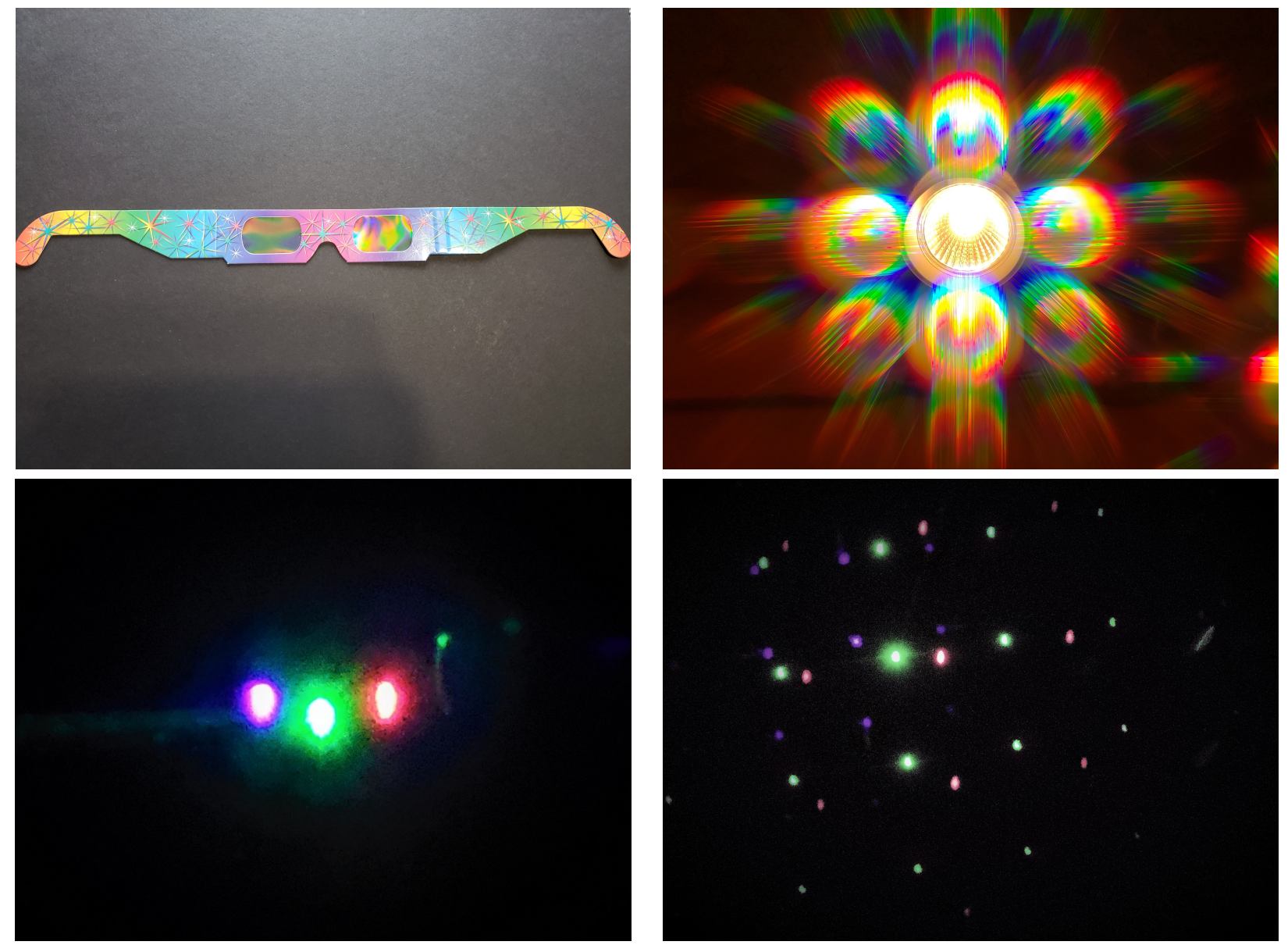

Figure 1. Diffraction glasses are used to separate the white light into their components. In the case of white light, the components are a continuum due to the nature of this kind of light. In the case of laser, a monochromatic source, the result is a grid caused by the gratings.

This warm-up activity can be used to ask fundamental questions like the order of the colors in the grid, the difference between the white source and the laser. This activity can be complemented showing the table or an 
image of a rainbow or both depending on the age. This warm-up activity can take much time, mainly because the kids spent time exploring, chatting, sometimes taking pictures and asking questions.

\subsection{Phosphorescence and fluorescence}

In our region, the phenomenons phosphorescence and fluorescence are known but commonly mistaken, although both are a type of luminescence, both terms are used indistinctly. This misconception was identified as soon as this activity was presented the first time, even professors and physics students use the terms indistinctly.

The first part of this activity is exploration; a low light condition is required. Using a phosphorescent material, usually we use t-shirts but can be replaced with plastic figures or paper, and a fluorescent material like a highlighter or paper, the paper is preferred cause can be used as a screen. The materials are illuminated with white or UV light, UV light preferably, the results of the experiment are shown in Figure 2.
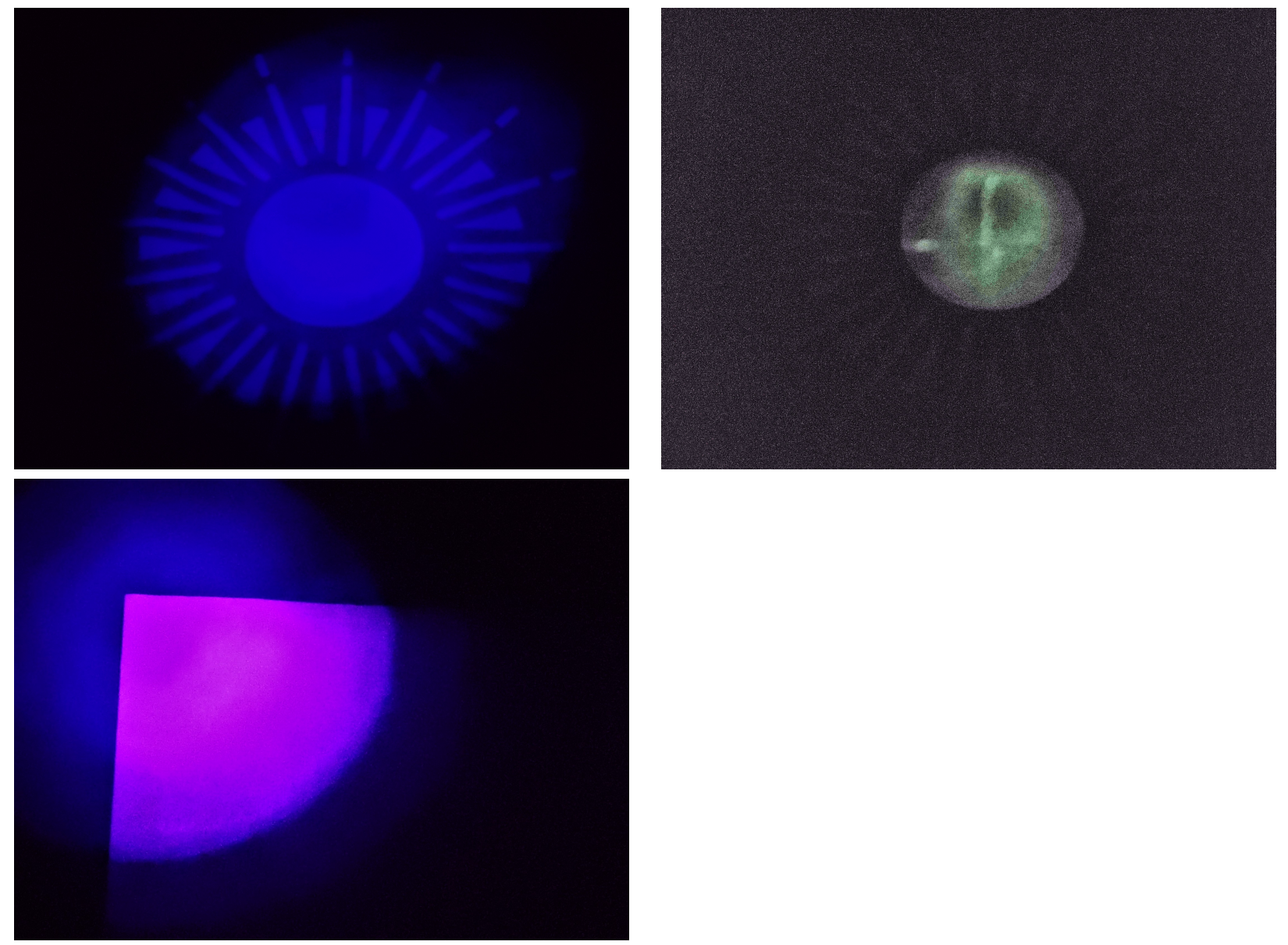

Figure 2. Luminescence phenomena. The Phosphorescence does not re-emit light immediately like in fluorescence. In most cases, the materials re-emit light on longer wavelengths or lower energy. In the first picture, a UV light is over a phosphorescent material in the next picture the light is turned off, and the material keeps glowing. The third picture shows a fluorescence paper if we turn off the UV light the picture will be black. It is difficult to take pictures in low light conditions.

This activity leads to fundamental questions about what is the difference in both materials. The kids will see that the phosphorescent material glows even without light and the fluorescent material shine only under the light. Usually, they have known these phenomena.

The kids understand that energy is absorbed by the materials and re-emitted, in the case of phosphorescence, gradually, and in the case of fluorescence rapidly. However, the fluorescence has a more vivid color. It is essential 
to remark in this activity the term energy. In our country, the law of conservation of energy is known by secondary school students and remarked in this activity.

The first exploration leads to other experiments because the kids can explore with materials they carry; usually, they light up their clothes like shoes or a highlighted book.

\subsection{Lesson}

At this point, these 2 activities are for warm-up, the kids explore materials, the electromagnetic spectrum, and both luminescent phenomena. The objective of the lesson is to explore energy levels for this is essential that in the first activity we remark that violet light has higher energy and can be done using common knowledge. The general example is that sometimes we are recommended to use sunscreen which protects us from Ultra Violet light, remarking the word Violet. This example is a piece of common knowledge in our city. Then, if the UV light is dangerous because it can cause burns, the red light is the least dangerous of all the colors, even remote controls have infrared light. Moreover, in the second activity, we remark the law of conservation of energy for secondary school kids or only that materials absorb energy.

At this point, we can combine both activities but first, in our country another misconception is about the laser light. It is believed that laser, no matter the color, is the strongest light of all. We shine the phosphorescent material with the three lasers as seen in the first picture of Figure 3. The first common question asked at this point is why the red laser, being the laser the strongest light of all, did nothing to the material. The result of this experience is shocking for most kids. At this point, they can sort the light by color, violet, the strongest, green not so strong and red the weakest of the three lasers. Even if at this point, they only sort the colors, they are relating the colors with energy, and we remark this concept.
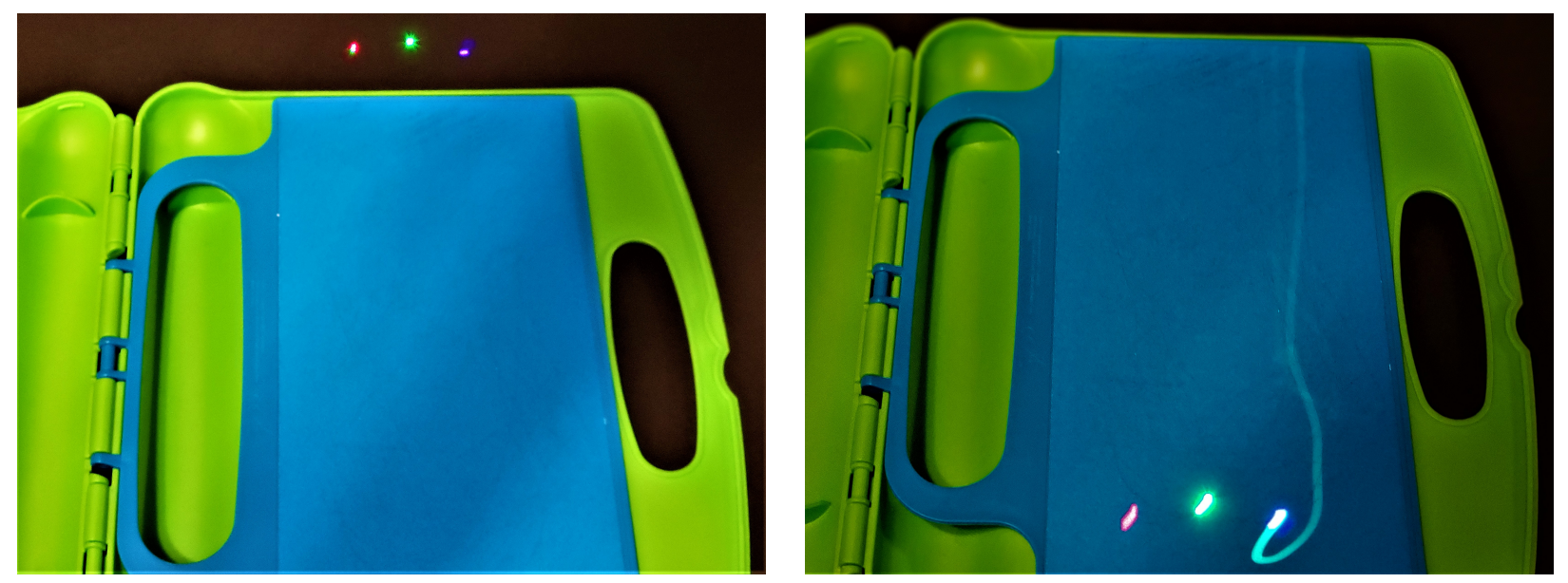

Figure 3. Three lasers are pointed to a phosphorescent material. In this case, only the violet laser produces the effect. In other papers, the green can produce the effect too. The color with the highest energy is the violet, and the paper can absorb this energy a re-emit it in other colors with lower energy.

The next activity is to combine the fluorescent material with the lasers. We shine the other material with the three lasers as seen in Figure 4. Another unexpected thing happens in this activity, now the green and violet laser change its color, but the red has no change, in this case, the green change color to a yellowish and violet to an apparent white. Kids usually expect the change for the violet color due to the past activity, but not from the green color.

This point is excellent to show the table or the rainbow again, now they identify the colors, being below the violet all other colors, below green only yellow, orange and red colors, and red have no colors below.

Now, to fully understand why the laser color changed, diffraction glasses are handed again. Now shine the fluorescent material with a green laser, the kids will now see not only the grid of points, but a continuum of colors that have not been there before, and the green is at the top of these colors. Now shine the material with 
the violet laser, and other colors will appear, and again the violet will be at the top of all colors. This is shown in the bottom pictures of Figure 4.

For secondary school students, the law of conservation of energy is the key to understand these phenomena. The material absorbs light with high energy like the violet and then re-emit light with lower energy or other colors. In the case of the green laser, the material absorbs it a re-emit light with lower energy like yellow, orange or red, but never higher energy like blue or violet. The same happens with the phosphorescent material, but the glasses are not required. The yellowish phosphorescent material can absorb light with high energy like green or violet, but not from lower energy like orange or red, that is why the material can never glow with the red laser.
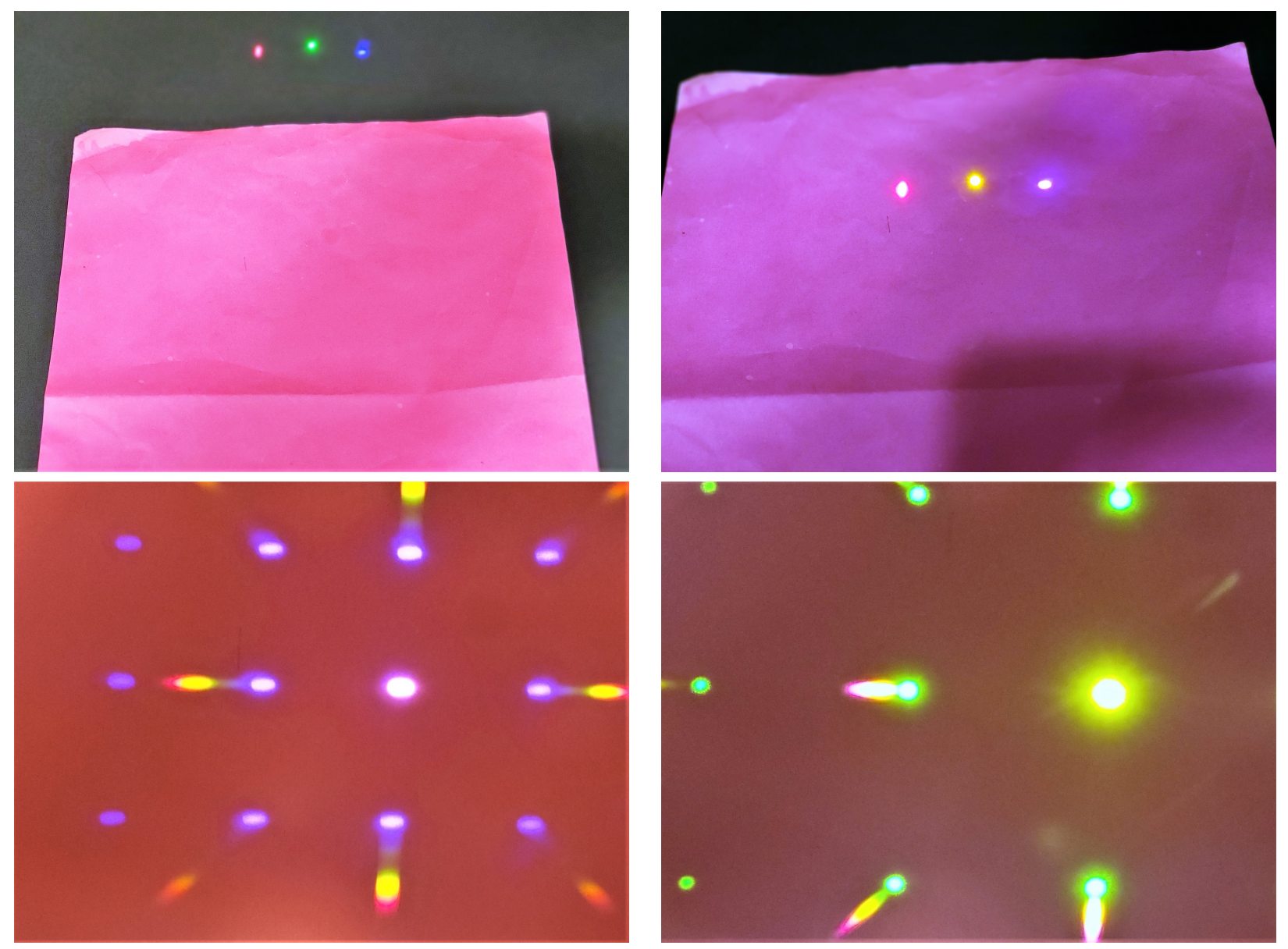

Figure 4. Fluorescence also is a reemission of light, but in this case, is faster than in the phosphorescence. The paper absorbs the energy from violet and green pointers and then is re-emitted by the paper. The highest color that can appear in both cases is the color of the laser due to the law of conservation of energy in this case.

\subsection{Discussion}

This lesson is usually performed in schools by our outreach group. It has been a great lesson since it has components teachers in our country wants because it has exploration, introduction to new concepts, experimentation affordable materials and tries to manage the misconceptions. The kids are amazed by the activities of this lesson, by the first activity they are engaged and usually ask several questions, or they want to explore with the materials. On the other hand, this lesson has materials light and easy to move, the duration can be adjusted because sometimes the schools change the schedule.

A few downsides have been detected for this lesson. The first, diffraction glasses are expensive and hard to find in our country. Also, sometimes the kids have questions out of the reach of this activity like, how the 
glasses work, or what are the specific components of the luminescent materials. In a few occasions, the low light conditions of the room were not meet and make the lesson difficult.

\section{ACKNOWLEDGMENTS}

Thanks to the Facultad de Ciencias Físico Matemáticas for the support. Also, thanks to SPIE for all the support, especially for the diffraction glasses which are difficult and expensive to obtain in our México. Also, thanks to all the volunteer students involved in the program Física Pato2, since they presented this activity in schools and gave feedback about how to improve it.

\section{REFERENCES}

[1] Malacara, D., [Color Vision and Colorimetry: Theory and Applications, Second Edition], SPIE (aug 2011).

[2] Perla M. Viera-González, G. E. S.-G., "Optics 4 every1, the hands-on optics outreach program of the universidad autonoma de nuevo leon," 9946 (2016).

[3] Perla Marlene Viera-González, G. E. S.-G., "Idl in monterrey: science, art and culture of light," 10741 (2018). 